\title{
Clinical consult column
}

\section{Dear Editor}

I am writing about a 64-year-old gentleman who had a left hemisphere CVA. He was hospitalized on our acute rehabilitation unit about 2 weeks ago and is expected to stay another 3-4 weeks. In the first days after his admission he was described as restless and agitated. Twice during the course of physical therapy he struck out at staff. Although he hasn't tried to hit anyone since, he does have fits of yelling. Yelling is especially bad when we're doing a range of motion exercises. I'm afraid this gentleman is making very little progress. Staff avoid him whenever possible. The other patients avoid him too. Whenever he raises his voice or appears to be upset they take him back to his room. He acts depressed much of the time. The doctors on our team are concerned about giving him medication because he's lethargic and older than many of our patients. What do you recommend, and please consider recommendations for interdisciplinary staff? We're hoping to discharge him to home, especially if we can teach him to transfer more independently.

\section{S.K.}

Cleveland, Ohio

\section{Dear S.K.}

In response to your question regarding your 64-year-old patient who had a left hemisphere cerebrovascular accident, I have the following comments. Information on the etiology of the stroke, specifically, embolic, thrombotic or hemorrhagic and associated neuropathologic findings, based on imaging studies, would be help- ful in understanding the current neurobehavioral manifestations.

There is clearly the suggestion that this patient is having pain that should be addressed aggressively. Shoulder pain, secondary to biomechanical stressors and traction capsulitis is quite common in the post-CVA population. Have intra-articular injections of local anesthetic and steroid followed by mobilization of the shoulder been considered? Additionally, have you ruled out such post-stroke conditions as heterotopic ossification and reflex sympathetic dystrophy that may, at least in part, be pain generators?

If all the aforementioned potential sources of pain have been ruled out and we are simply dealing with agitation, then I would recommend consideration of psychopharmacological treatment with one or a combination of 'anti-aggression' agents potentially including selective serotonergic re-uptake inhibitors (SSRIs), buspirone, psychotropic anticonvulsants such as carbamazepine or valproic acid and/or lithium carbonate. It is important to remember that patients with severe receptive dysphasia may demonstrate significant paranoid-like behavior, sometimes in conjunction with agitation, due simply to their inability to understand and communicate. That is, they will often misinterpret information leading to further confusion and exacerbation of their agitation.

Affectively, if the patient is depressed, he may simply have a so-called agitated depression which is not uncommon in the geriatric population. If this is the case then treatment of his depression through both pharmacologic and non-pharmaco- 
logic modalities would be indicated. Lethargy in and of itself is by no means a contraindication to medicating a patient. The internal physiologic milieu, as well as, the external environmental milieu need to be looked at in terms of assuring that there are no correctable etiologies for the patient's lethargy and/or agitation. If the patient has an occult infection, drug side effect, or electrolyte and/or hormonal abnormality, these might all play a role in his observed lethargy and would need to be treated if they were present. Always remember the ABCs of behavioral analysis: antecedents of behavior, how the behavior is manifested, and the consequences of the behavior. By keeping these three facts in mind you will optimize your ability to deal with behavioral issues that potentially interfere with ongoing rehabilitation efforts.

Nathan D. Zasler, Editor 